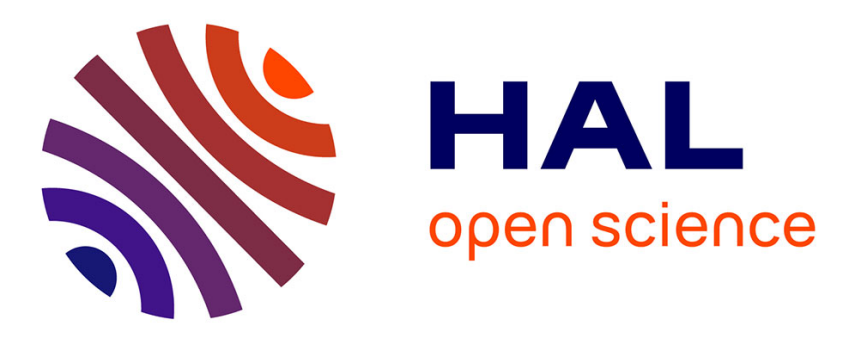

\title{
Régimes transitoires d'écoulements newtoniens et pseudoplastiques
}

\author{
X. de Saint Victor, D.P. Ly, D. Bellet
}

\section{To cite this version:}

X. de Saint Victor, D.P. Ly, D. Bellet. Régimes transitoires d'écoulements newtoniens et pseudoplastiques. Revue de Physique Appliquée, 1981, 16 (5), pp.217-223. 10.1051/rphysap:01981001605021700 . jpa-00244914

\section{HAL Id: jpa-00244914 https://hal.science/jpa-00244914}

Submitted on 1 Jan 1981

HAL is a multi-disciplinary open access archive for the deposit and dissemination of scientific research documents, whether they are published or not. The documents may come from teaching and research institutions in France or abroad, or from public or private research centers.
L'archive ouverte pluridisciplinaire HAL, est destinée au dépôt et à la diffusion de documents scientifiques de niveau recherche, publiés ou non, émanant des établissements d'enseignement et de recherche français ou étrangers, des laboratoires publics ou privés. 


\title{
Régimes transitoires d'écoulements newtoniens et pseudoplastiques
}

\author{
X. de Saint Victor, D. P. Ly et D. Bellet \\ Institut de Mécanique des Fluides, 2, rue Camichel, 31071 Toulouse Cedex, France \\ (Reçu le 9 décembre 1980, révisé le 26 janvier 1981, accepté le 3 février 1981)
}

\begin{abstract}
Résumé. - On résout théoriquement et numériquement le problème des démarrages d'écoulements de fluides à comportement netwtonien ou pseudoplastique. Les gradients de pression à l'origine des écoulements sont soit constants soit fonction du temps.

Une méthode expérimentale d'étude est aussi proposée. Les résultats présentés montrent l'influence de divers paramètres rhéologiques, hydrodynamiques ou géométriques sur la durée de tels régimes transitoires d'écoulement.

Abstract. - The effects of rheological, hydrodynamic and geometrical parameters are examined by solving the Navier-Stokes equations by using different mathematical techniques with regard to start-up flows.

An experimental method is therefore proposed. The flows are subjected to a constant pressure gradient or to an unsteady one. The fluids studied exhibit either newtonian or pseudoplastic behaviour. The results presented here show the influence of different parameters upon the duration of this start-up.
\end{abstract}

\section{Notations :}

$\rho A$ amplitude du gradient de pression,

$K$ consistance,

$n$ indice de comportement,

$r$ position radiale,

$R$ rayon du tube.

$t$ temps,

$t^{*} \quad$ temps réduit,

$u$ vitesse longitudinale,

$u^{*} \quad$ vitesse réduite,

$\bar{u} \quad$ vitesse moyenne.

Symboles grecs :

$\xi \quad$ position radiale adimensionnelle,

$\rho$ densité,

$\tau_{r z} \quad$ contrainte de cisaillement,

$\omega$ pulsation.

1. Introduction. - Les problèmes liés à la mise en mouvement d'écoulements newtoniens en conduites cylindriques circulaires sont d'une résolution relativement aisée du fait de la linéarité de l'équation du mouvement régissant le phénomène. Ceci explique sans doute le fait que la majeure partie des publications traitant des régimes transitoires concerne l'étude d'écoulements newtoniens généralement soumis, par ailleurs, à un gradient de pression constant.
Les premiers travaux concernant ce problème semblent avoir été réalisés par Gromecka, dès 1882 [1]. Cinquante ans plus tard, Szymanski étendait le domaine de validité de la solution de Gromecka en considérant des gradients de pression non permanents [2].

Dans le cas de fluides à comportement non newtonien, le problème a été moins abordé et la plupart des auteurs considèrent alors un gradient de pression constant. C'est le cas de deux publications qui traitent $\mathrm{du}$ problème en écoulement pseudoplastique. La première est proposée par Sestak et Charles [3] qui utilisent une méthode développée par Starg et Slyoskin [4] pour l'étude de certains phénomènes newtoniens.

Edwards, Nellist et Wilkinson étudient, quant à eux, les écoulements pulsés de fluides pseudoplastiques par une méthode numérique et mentionnent accessoirement le problème du régime transitoire [5].

Les principaux travaux sont référencés sur la figure 1 et les formes des gradients de pression utilisés sur la figure 2 .

2. Etude théorique. - L'équation du mouvement régissant le problème du régime transitoire en conduite d'un fluide incompressible est :

$$
\rho \frac{\partial u}{\partial t}(r, t)=-\frac{\partial p}{\partial z}+\frac{1}{r} \frac{\partial}{\partial r}\left(r \tau_{r z}\right)
$$




\begin{tabular}{|c|c|c|c|}
\hline AUTEUR & NEWTONIEN & $\begin{array}{c}\text { NON } \\
\text { NEWTONIEN } \\
\text { INELASTIQUE }\end{array}$ & $\begin{array}{l}\text { VISCO- } \\
\text { ELASTIOUUE }\end{array}$ \\
\hline GROMECKA (1882) & 太 & & \\
\hline SZYMANSKI (1932) & $\star$ & & \\
\hline ATABEK (1962) & $\star$ & & \\
\hline ATABEK $\quad$ (1964) & & $\star$ & \\
\hline ETTER (1965) & & & $\star *$ \\
\hline SESTAK (1968) & & $\hbar *$ & \\
\hline AVULA (1969) & $\star 0$ & & \\
\hline DOWNING (1971) & $\star$ & & \\
\hline WATERS (1971) & & & $\lambda$ \\
\hline AVULA $\quad$ (1971) & $* 0$ & & \\
\hline DUGGINS (1972) & & * & \\
\hline EDWARDS (1972) & & 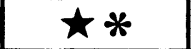 & \\
\hline DUGGINS (1972) & & $*$ & \\
\hline KATAOKA (1975) & 00 & & \\
\hline MASLIYAH (1975) & $\star 0$ & & \\
\hline I.M.F.T. & $\star 00$ & & \\
\hline $\begin{array}{l}\text { Contribution } \\
\text { personnelie }\end{array}$ & $\star * 00$ & $\star * 00$ & \\
\hline$\downarrow$ Méthode analyti & & $\begin{array}{l}\text { betermination e } \\
\text { radient de pre }\end{array}$ & $\begin{array}{l}\text { xpérimentale } \\
\text { ssion }\end{array}$ \\
\hline
\end{tabular}

Fig. 1. - Principaux travaux. Méthodes de résolution.

[Main works. Methods of resolution.]

il convient d'y ajouter la condition initiale et les conditions aux limites suivantes :

$$
\begin{aligned}
u(r, 0) & =0, \quad \forall r \\
u(R, t) & =0, \quad \forall t \\
\left.\frac{\partial u}{\partial r}\right|_{r=0} & =0, \quad \forall t
\end{aligned}
$$

2.1 Cas newtonien. - Dans le cas newtonien, l'équation (1) s'écrit :

$$
\rho \frac{\partial u(r, t)}{\partial t}=-\frac{\partial p}{\partial z}+\frac{1}{r} \frac{\imath}{\partial r}\left(r \eta \frac{\partial u}{\partial r}\right)
$$

ou encore, sous forme adimensionnelle :

$$
\frac{\partial^{2} u^{*}}{\partial \xi^{2}}+\frac{1}{\xi} \frac{\partial u^{*}}{\partial \xi}-\mathscr{R}_{\mathrm{e}}^{*} \frac{\partial u^{*}}{\partial t^{*}}=-8 \tilde{f}\left(t^{*}\right)
$$

où :

$$
\begin{gathered}
-\frac{\partial p}{\partial z}=\rho A . f(t) ; \quad f\left(T t^{*}\right)=\tilde{f}\left(t^{*}\right) ; \\
t^{*}=\frac{t}{T}=\frac{\omega t}{2 \pi} ; \quad \xi=\frac{r}{R}
\end{gathered}
$$

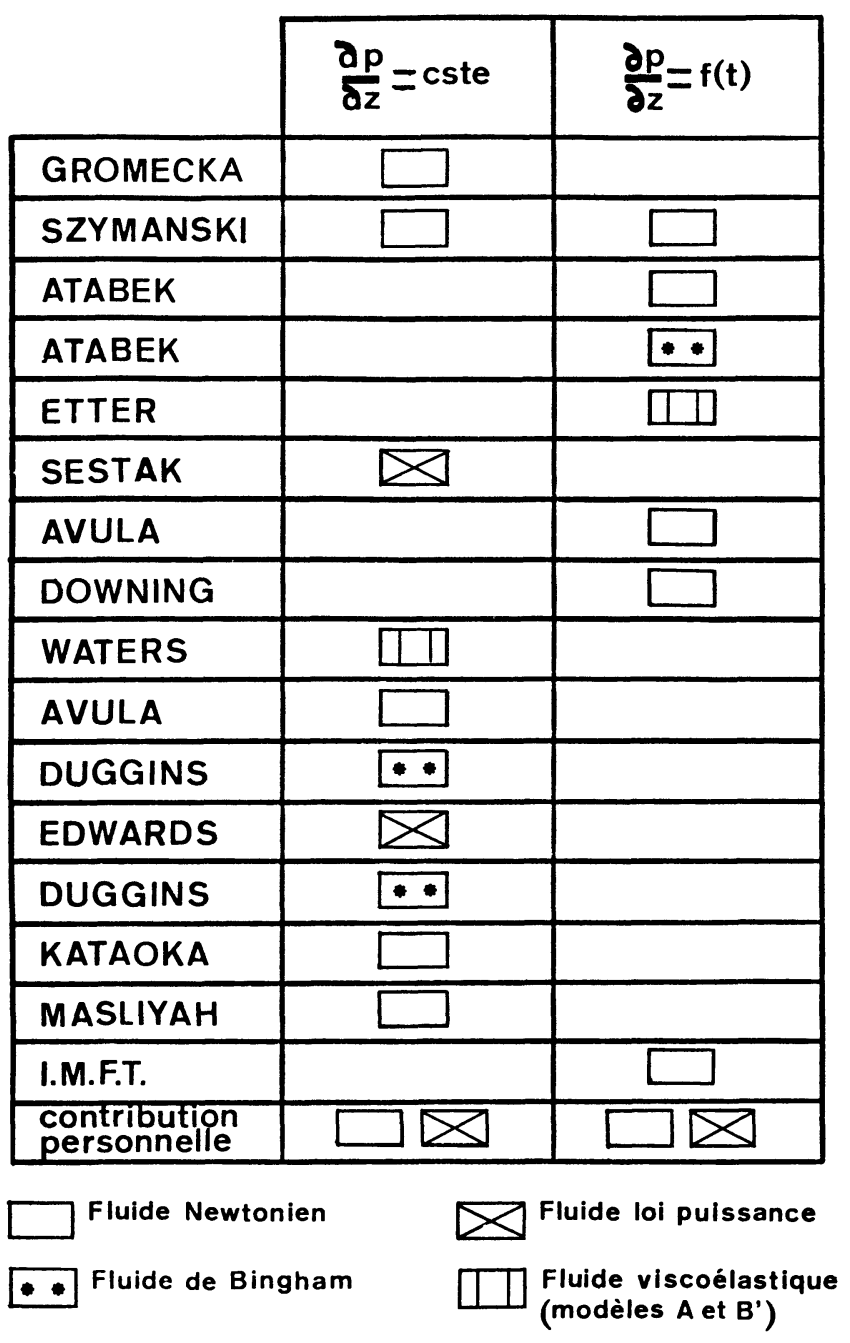

Fig. 2. - Forme du gradient de pression.

[Pressure gradient form.]

$$
\begin{gathered}
u^{*}=\frac{u}{\bar{u}}, \quad \text { avec }: \quad \bar{u}=\frac{A R^{2}}{8 v} \\
\mathscr{R}_{\mathrm{e}}^{*}=\frac{\rho \omega R^{2}}{2 \pi \eta}
\end{gathered}
$$

nombre égal à un facteur près au nombre de Womersley : $\alpha^{2}=\rho \omega R^{2} / \eta$.

L'équation (6) peut être résolue en utilisant la méthode de transformation de Laplace.

On a :

$$
\begin{gathered}
U(\xi, s)=\int_{0}^{1} \mathrm{e}^{-s t^{*}} u^{*}\left(\xi, t^{*}\right) \mathrm{d} t^{*} \\
F(s)=\int_{0}^{\infty} \mathrm{e}^{-s t^{*}} \tilde{f}\left(t^{*}\right) \mathrm{d} t^{*}
\end{gathered}
$$

où $s$ est le paramètre symbolique.

L'équation (6) peut donc s'écrire :

$\frac{\mathrm{d}^{2}}{\mathrm{~d} \xi^{2}}[U(\xi, s)]+\frac{1}{\xi} \frac{\mathrm{d}}{\mathrm{d} \xi}[U(\xi, s)]-$

$$
-\mathscr{R}_{\mathrm{e}}^{*} . s . U(\xi, s)=-8 F(s) .
$$


Cette équation est du type équation de Bessel. Sa solution satisfaisant aux conditions aux limites énoncées précédemment est :

$$
U(\xi, s)=\frac{8}{\mathscr{R}_{\mathrm{e}}^{*}} \cdot \frac{1}{s}\left[1-\frac{J_{0}\left(i \sqrt{\mathscr{R}_{\mathrm{e}}^{*} s} \cdot \xi\right)}{J_{0}\left(i \sqrt{\mathscr{R}_{\mathrm{e}}^{*} s}\right)}\right] F(s) .
$$

L'application du théorème de convolution et du théorème des résidus permet d'obtenir finalement :

$$
\begin{aligned}
& u^{*}\left(\xi, t^{*}\right)=\frac{16}{\mathscr{R}_{\mathrm{e}}^{*}} \sum_{n=1}^{\infty} \frac{J_{0}\left(a_{n} \zeta\right)}{a_{t_{n}} J_{\mathrm{1}}\left(a_{n}\right)} \times \\
& \times \int_{0}^{t^{*}} \tilde{f}\left(t^{*}-t^{\prime}\right) \exp \left(-\frac{1}{\mathscr{R}_{\mathrm{e}}^{*}} a_{n}^{2} t^{\prime}\right) \mathrm{d} t^{\prime} .
\end{aligned}
$$

2.2 Cas non newtonien. - Dans le cas ostwaldien, l'équation (1) s'écrit :

$$
\rho \frac{\partial u(r, t)}{\partial t}=-\frac{\partial p}{\partial z}+\frac{1}{r} \cdot \frac{\partial}{\partial r}\left(r K \frac{\partial u}{\partial r}\left|\frac{\partial u}{\partial r}\right|^{n-1}\right) .
$$

La non-linéarité de cette équation ne permet pas d'obtenir, comme dans le cas précédent, une solution analytique. Nous proposons ici deux méthodes de résolution par approximation.

2.2.1 Méthode $n^{0}$ 1. - La première de ces méthodes est une méthode aux différences finies, du premier ordre. Le schéma utilisé est un schéma à trois points. L'équation (12) sous forme adimensionnelle est écrite sous forme d'une équation aux différences finies. Le maillage choisi est explicité figure 3 .

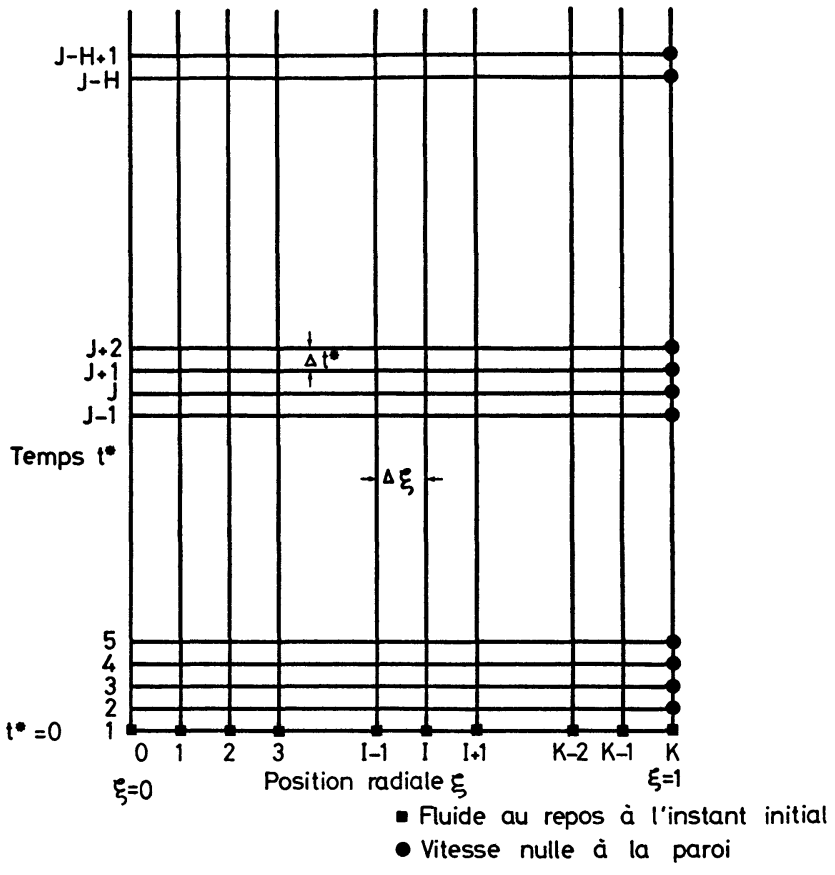

Fig. 3. - Méthode aux différences finies. Maillage choisi.

[Finite difference network.]

Le rayon de la conduite est décrit de $I=1$, valeur correspondant à l'axe du tube, à $I=K$ correspondant à la paroi, avec un pas $\Delta \xi=0,05$.

La seconde variable $t^{*}$ est discrétisée de $J=1$, correspondant à l'état initial, à $J=L$ correspondant à un instant ultérieur au régime transitoire. Le pas $\Delta t^{*}$, choisi en fonction des valeurs données aux différents paramètres, est généralement de l'ordre de $10^{-3}$.

Les équations sont :

$$
\begin{aligned}
& u_{I, J}^{*}=u_{I, J-1}^{*}+\frac{\Delta t^{*}}{\beta^{2}}\left\{N f\left(t^{*}\right)+\frac{1}{\xi \Delta \xi^{n+1}}\left[\left(\xi+\frac{\Delta \xi}{2}\right)\left(u_{I+1, J}^{*}-u_{I, J}^{*}\right)\left|u_{I+1, J}^{*}-u_{I, J}^{*}\right|^{n-1}-\right.\right. \\
& \left.\left.-\left(\xi-\frac{\Delta \xi}{2}\right)\left(u_{I, J}^{*}-u_{I-1, J}^{*}\right)\left|u_{I, J}^{*}-u_{I-1, J}^{*}\right|^{n-1}\right]\right\} \\
& u_{1, J}^{*}=u_{1, J-1}^{*}+\frac{\Delta t^{*}}{\beta^{2}}\left\{N f\left(t^{*}\right)+\frac{4}{\Delta \xi^{n+1}}\left(u_{2, J}^{*}-u_{1, J}^{*}\right)\left|u_{2, J}^{*}-u_{1, J}^{*}\right|^{n-1}\right\} \\
& \text { où : } \quad \beta^{2}=\frac{\rho \omega \bar{u}^{1-n} R^{n+1}}{2 \pi K} \quad \text { et } \quad N=2 \cdot\left(\frac{1+3 n}{n}\right)^{n} \\
& \bar{u}=\frac{n}{3 n+1} \cdot \frac{R^{1+!}}{(2 K)^{1 / n}}(\rho A)^{1 / n} .
\end{aligned}
$$

2.2.2 Méthode $n^{0}$ 2. - La deuxième solution proposée consiste à remplacer le terme accélération figurant dans l'équation (12) par l'intégrale de sa valeur moyenne le long du rayon de la conduite (Cf. [3]) :

$$
\frac{\partial u}{\partial t} \simeq \frac{1}{R} \int_{0}^{R} \frac{\partial u}{\partial t} \mathrm{~d} r=\varphi(t)
$$

Cette approximation permet d'écrire avec :

$$
\begin{gathered}
V(\xi, t)=\frac{u(\xi, t)}{\bar{u}} \\
V(\xi, t)=\frac{n}{n+1}\left(\frac{N}{2 A}\right)^{\frac{1}{n}}\left(\xi^{1+\frac{1}{n}}-1\right) \chi(t)
\end{gathered}
$$




$$
\begin{aligned}
& \text { où : } \chi(t)=[\varphi(t)-A f(t)]|\varphi(t)-A f(t)|^{\frac{1}{n}-1} \\
& \text { et } \frac{\mathrm{d} \chi(t)}{\mathrm{d} t}=-\frac{2 n+1}{n}\left(\frac{2 A}{N}\right)^{1 / n} \times \\
& \times \frac{1}{\bar{u}}\left[A f(t)+\chi(t)|\chi(t)|^{n-1}\right] \text {. }
\end{aligned}
$$

Les solutions de l'équation (16) et de l'équation (18) sont obtenues numériquement par une méthode de Runge-Kutta.

3. Méthode expérimentale. - Le fluide étant initialement au repos, le modèle expérimental permet de lui appliquer, à un instant donné, un gradient de pression constant, oscillatoire ou pulsé. L'installation comporte 4 parties principales (Fig. 4) :

- un tube rigide,

- une pompe à vitesse variable produisant des écoulements permanents,

- un système pulseur,

- un système de synchronisation des démarrages permettant de démarrer l'écoulement avec toujours la même valeur du gradient de pression.

Le tube rigide en plexiglas est placé horizontalement. Sa transparence permet de visualiser l'écoulement et de mesurer les vitesses locales par vélocimétrie à laser. Les fluides utilisés sont soit des solutions

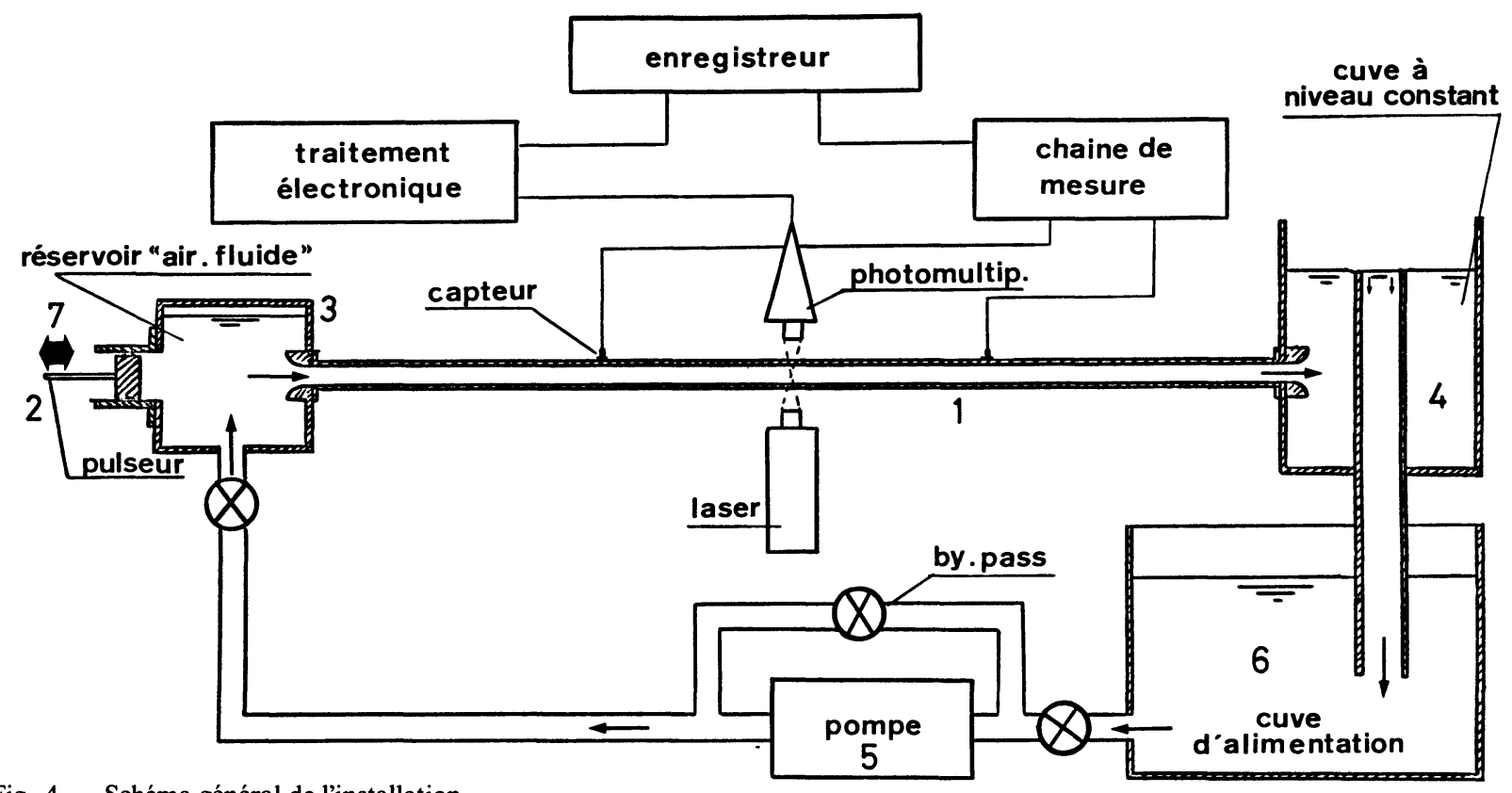

Fig. 4. - Schéma général de l'installation.

[General diagram of the experimental device.]

aqueuses de Pluracol V10 à comportement newtonien, soit des solutions aqueuses de carboxyméthylcellulose de sodium ( $\mathrm{Na} \mathrm{CMC}$ ) dont le comportement est nettement pseudoplastique.

Les profils de vitesse sont déterminés par un suiveur de fréquence. L'adjonction de cellules de Bragg permet la mesure des faibles vitesses et de détecter le sens de l'écoulement.

Le gradient de pression expérimental est obtenu à l'aide de capteurs de pression de type piézorésistif, placés sur le tube du modèle expérimental. Un exemple d'établissement du gradient de pression est donné sur la figure 5. On y remarque les faibles différences qu'il y a entre la forme expérimentale et la solution analytique associée.

4. Résultats. - Les résultats concernent l'influence des paramètres rhéologiques et géométriques sur la durée du temps d'établissement. Ce temps est défini comme celui au bout duquel le débit d'écoulement devient égal à $99 \%$ de sa valeur finale.

4.1 CAS NEWTONIEN. - Le paramètre rhéologique caractérisant l'écoulement newtonien est la viscosité dynamique $\eta$ dont nous avons étudié l'influence. On note que la durée du régime transitoire décroît quand augmente et tend vers l'infini quand la viscosité tend vers la valeur zéro (cas des fluides parfaits). Ces constatations sont d'autant plus nettes que le rayon de la conduite est plus faible (Fig. 6).

La figure 7 montre l'influence du rayon du tube. Il apparaît que la durée du régime transitoire croît avec le rayon. On vérifie les résultats obtenus précédemment, à savoir qu'à une valeur donnée du rayon, la durée d'établissement augmente quand la viscosité dynamique diminue.

D'après l'étude théorique, il s'avère que la fréquence de pulsation de l'écoulement n'intervient pas sur la 


$$
-\frac{\partial P}{\partial z}=290\left\{\left(1-e^{-0,8 t}\right)+0,63 \cos \pi(t-0,63)\left[1-e^{-0,8 t}\right]\right\}
$$

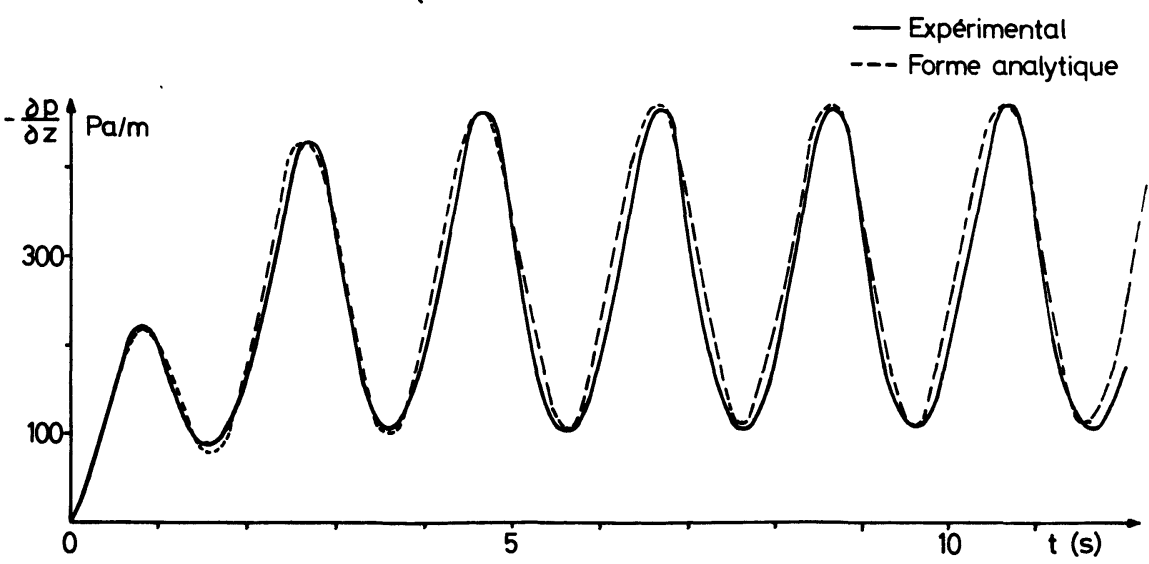

Fig. 5. - Etablissement d'un gradient de pression pulsatoire.

[Comparison between analytic and experimental forms of a pulsating pressure gradient.]

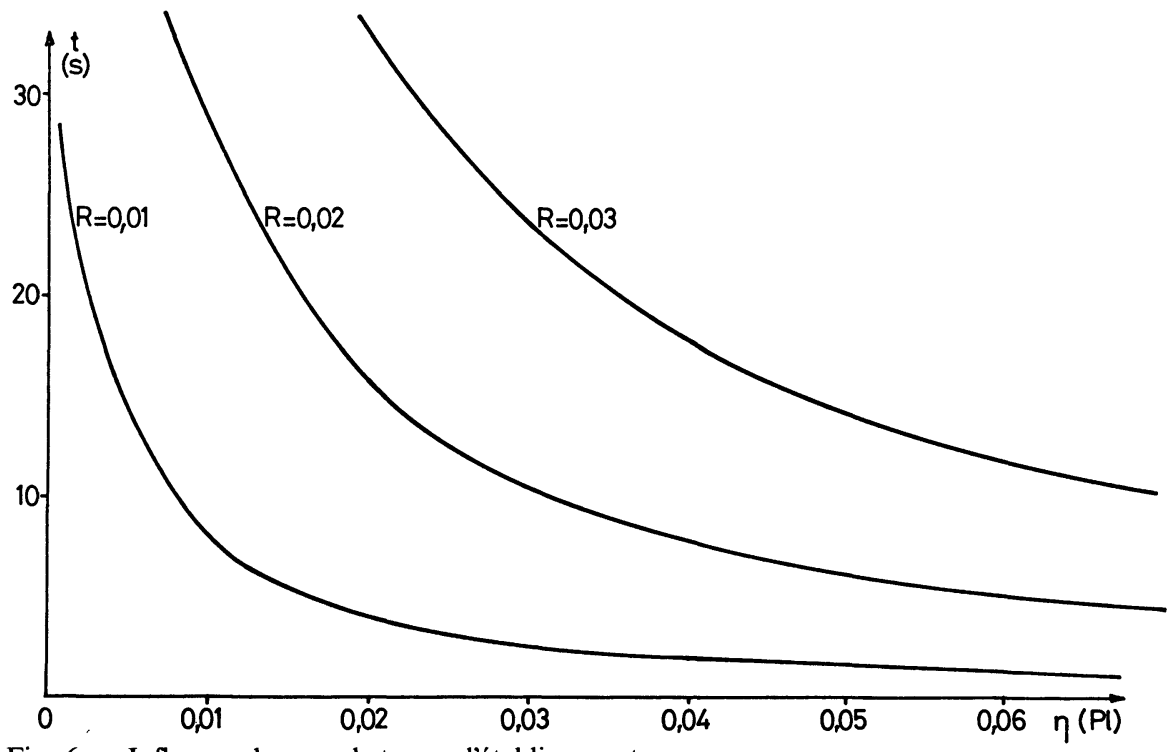

Fig. 6. - Influence de $\eta$ sur le temps d'établissement.

[Influence of $\eta$ upon the start-up time.]

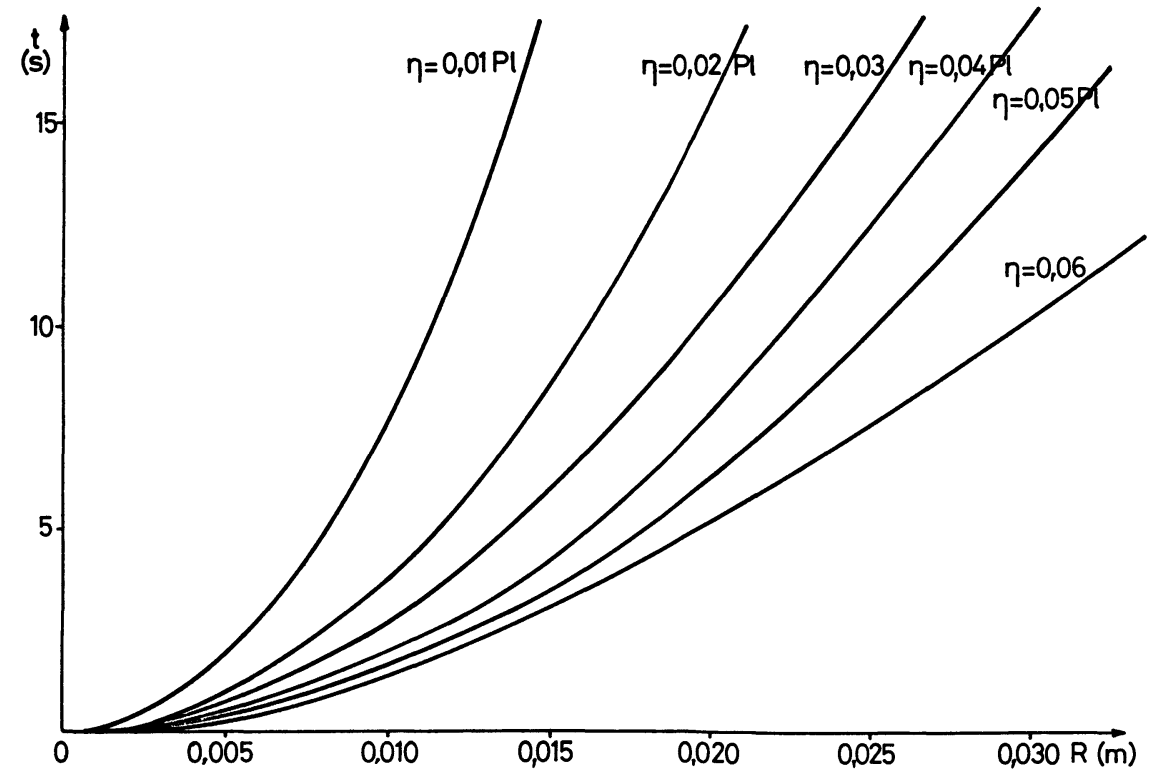

Fig. 7. - Influence de $R$ sur le temps d'établissement.

[Influence of $R$ upon the start-up time.] 
durée du régime transitoire; ceci est confirmé par l'expérience, ce qui permet d'assurer que les solutions aqueuses de Pluracol V10 ne présentent pas, pour des valeurs faibles de la fréquence, de caractère viscoélastique (Fig. 8).

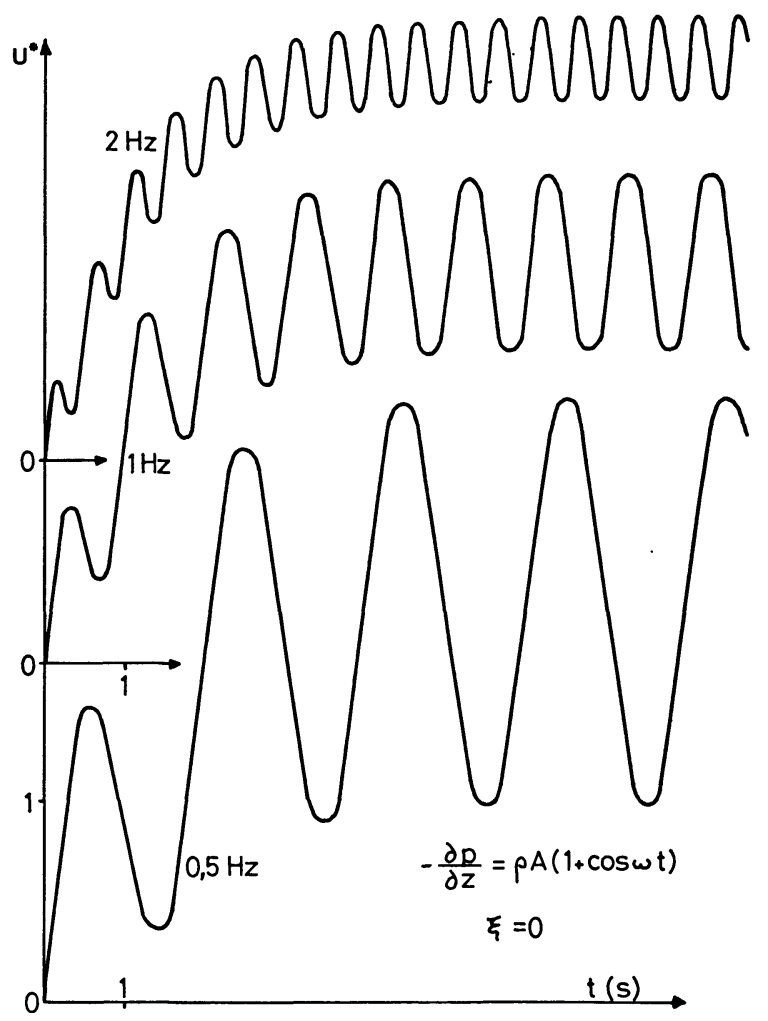

Fig. 8. - Cas newtonien. Influence de la fréquence.

[Newtonian case. Influence of the frequency.]

A la lumière de ces divers résultats, on peut remarquer l'importance du groupement $R^{2} / v$ qui peut être considéré comme étant une constante de temps régissant la durée du régime transitoire des écoulements newtoniens, comme on peut le remarquer sur la figure 9.

4.2 CAS NON NEWTONIEN. - Les deux paramètres rhéologiques gouvernant les écoulements ostwaldiens sont l'indice de comportement $n$ et la consistance $K$.

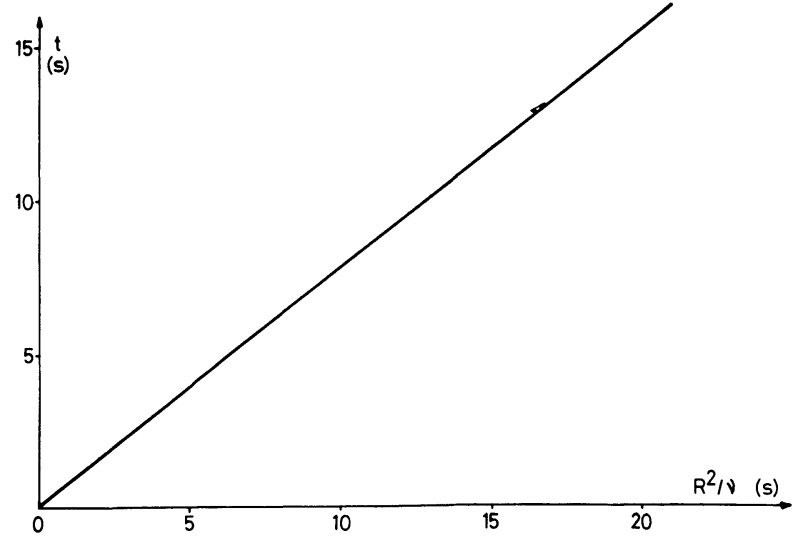

Fig. 9. - Influence du paramètre $R^{2} / v$ sur le temps d'établissement. [Influence of $R^{2} / v$ upon the start-up time.]
Nous avons étudié leurs effets sur l'établissement d'écoulements pseudoplastiques. Il est à noter que, dans les solutions de $\mathrm{NaCMC}$, nous ne pouvons faire varier $n$ indépendamment de $K$ et vice-versa. En effet, à chaque concentration de $\mathrm{Na}$ CMC dans l'eau, correspond un couple de valeur $(n, K)$.

Pour cette raison, les conclusions relatives aux influences de l'indice de comportement ou de la consistance sont issues des résultats obtenus par une des deux méthodes théoriques présentées.

Nous avons tout d'abord fait varier l'indice $n$ à $K$ constant. On observe que la durée du temps d'établissement augmente avec la pseudoplasticité. De plus, quand l'indice de comportement tend vers 1 , les différences de temps d'établissement, dues à des valeurs de $K$ différentes, sont moins nettes que pour des fluides de pseudoplasticité plus importante (Fig. 10).

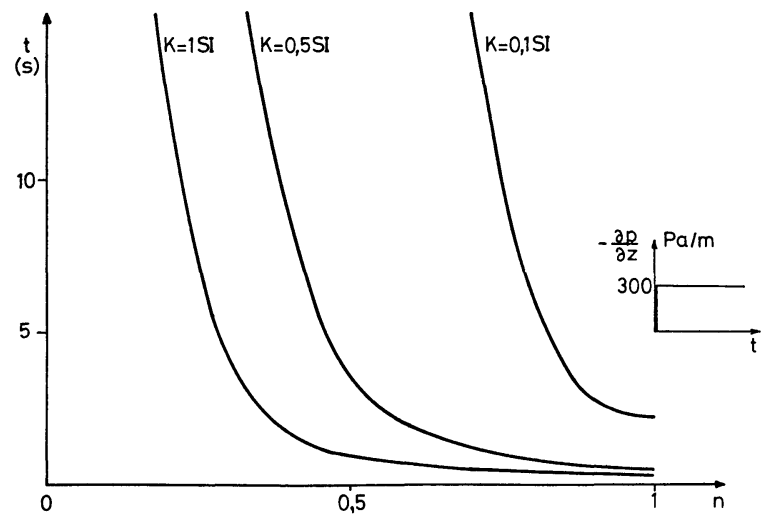

Fig. 10. - Influence de $n$ sur le temps d'établissement.

[Influence of $n$ upon the start-up time.]

Nous avons ensuite inversé le processus en faisant varier $K$ et en gardant $n$ constant. On remarque que le temps d'établissement diminue lorsque $K$ augmente (Fig. 11). On pouvait s'attendre à ce résultat qui corrobore celui déduit de la figure 6. En effet, quand $n$ vaut 1 la consistance $K$ peut être assimilée à une viscosité dynamique.

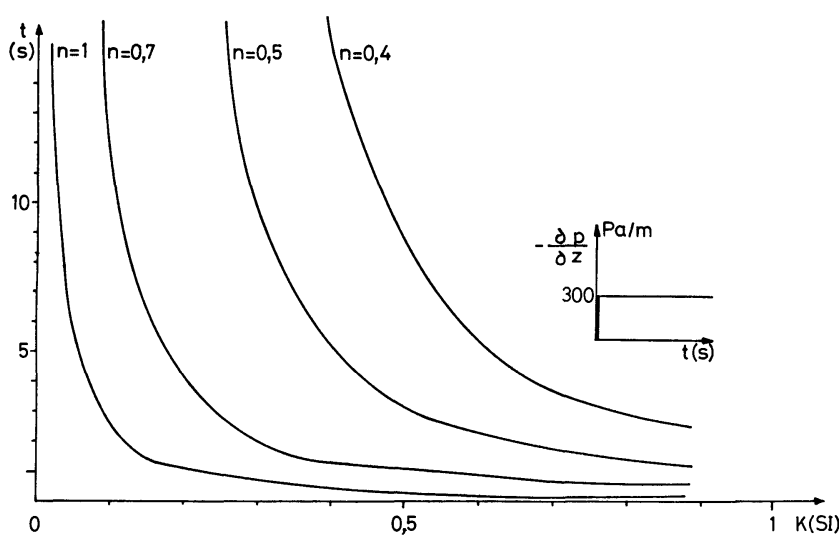

Fig. 11. - Influence de $K$ sur le temps d'établissement. [Influence of $K$ upon the start-up time.] 
Nous avons, d'autre part, étudié l'influence du rayon de la conduite pour trois couples de valeurs $n$ et $K$. On constate que, comme dans le cas newtonien, la durée du temps d'établissement varie dans le même sens que $R$, très lentement pour de petites valeurs de $R$ et beaucoup plus rapidement pour les grandes valeurs (Fig. 12).

Signalons enfin que, dans le cas d'un écoulement

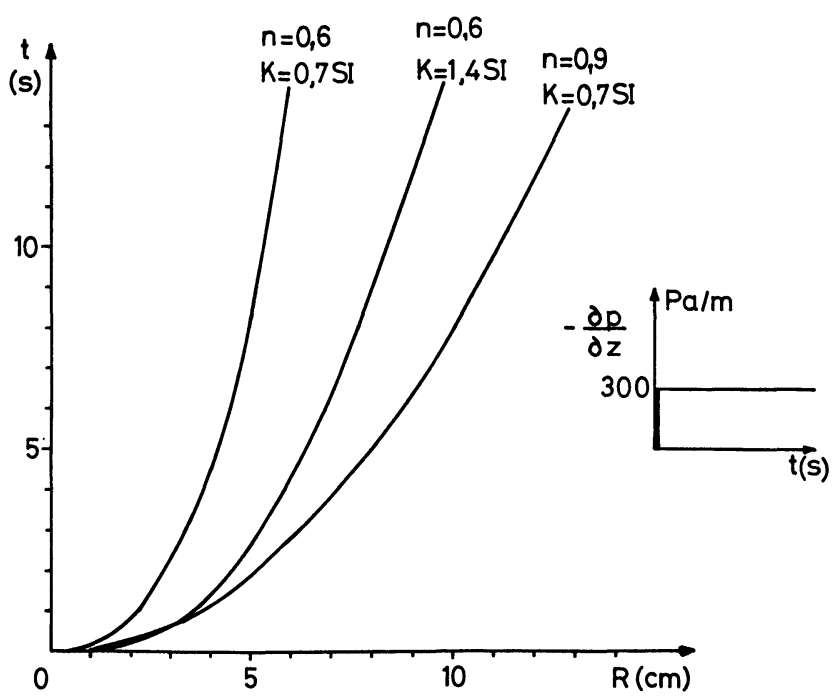

Fig. 12. - Cas non newtonien. Influence de $R$ sur le temps d'établissement.

[Non-newtonian case. Influence of $R$ upon the start-up time.] soumis à un gradient de pression pulsé, nous n'avons pas observé d'influence de la fréquence.

5. Conclusion. - La recherche systématique des influences des divers paramètres rhéologiques, hydrodynamiques ou géométriques sur l'établissement des régimes d'écoulement nous a amenés à un certain nombre de conclusions.

Dans le cas newtonien, on peut noter l'importance de la viscosité cinématique et du rayon de la conduite. De plus, rappelons la mise en évidence de la constante de temps $R^{2} / v$, dont la valeur est significative de la durée du temps d'établissement.

En ce qui concerne les écoulements pseudoplastiques, ce sont les paramètres rhéologiques $n$ et $K$ et la caractéristique géométrique $R$ qui paraissent être les plus influents sur le régime transitoire.

Par contre, lorsque le gradient de pression est pulsé, la fréquence et le taux de modulation ne semblent pas jouer de rôle significatif.

De ces résultats, on peut déduire que le temps d'établissement d'un régime d'écoulement, permanent ou non, est, en général, non négligeable, ce qui peut présenter une certaine importance sur le plan industriel ou dans des études d'hémodynamique par exemple.

De plus, nous avons vu que les paramètres rhéologiques jouaient un rôle essentiel dans l'évolution du régime d'écoulement. Ignorer ces paramètres dans l'évaluation d'un démarrage d'écoulement amènerait donc à commettre des erreurs importantes.

\section{Bibliographie}

[1] Gromecka, I. S., A contribution to the theory of viscous flow in narrow cylindrical tubes (en russe) (Kazanyi University Press), 1882.

[2] Szymanski, P., J. Maths Pures Appl. 11 (1932) 67.

[3] Sestak, J., Charles, M. E., Chem. Eng. Sci. 23 (1968) 1127.

[4] Slyoskin, N. A., TARg, S. M., Dokl. Akad. Nank., USSR 54 (1946) 27.

[5] Edwards, M. F., Nellist, D. A., Wilkinson, W. R., Chem. Eng. Sci. 27 (1972) 295.

[6] AtabeK, H. B., Zeit. Angew. Math. Mech. 44 (1964) 332.

[7] Etter, I., Schowalter, W. R., Trans. Soc. Rheol. 9 : 2 (1965) 351 .

[8] Atabek, H. B., Zeit. Angew. Math. Phys. 13 (1962) 417.

[9] Avula, X. J. R., J. Phys. Soc. Jpn 27 (1969) 497.

[10] Downing, C. G., AIChEJ 17 (1971) 322.
[11] Waters, N. D., King, M. J., J. Phys. 4 (1971) 204.

[12] Avula, X. J. R., Zeit. Angew. Math. Mech. 51 (1971) 517.

[13] Duggins, R. K., Chem. Eng. Sci. 27 (1972) 1991.

[14] DugGins, R. K., Int. Conf. on pressure surges (1972) BHRA Fluid Eng.

[15] Kataoka, K., Kawabato, T., Miki, K., Chem. Eng. Sci. 8 (1975) 277.

[16] Masliyah, J. H., Schook, C. A., Can. J. Chem. Eng. 53 (1975) 469.

[17] Ly, D. P., Bellet, D., Bousquet, A., Transient regimes of unsteady state flow of viscous fluids, Euromech 73, Aix-enProvence (1976).

[18] De Saint-Victor, X., Thèse de spécialité, 1979, Toulouse, France. 